TRABAJO EN PROGRESO

\title{
Gamificación para el aprendizaje de la apicultura
}

\author{
Luis Gerardo Castillo Pinkus, Víctor Hugo \\ Menéndez Domínguez, José Chavier De Araujo \\ Freitas, Yolanda Beatriz Moguel Ordóñez, Edith \\ Coello Cervera
}

Publicado: 31 Octubre 2018

\begin{abstract}
Resumen
Las abejas han sido declaradas recientemente como el organismo más importante del planeta, por ser polinizadores y generadores de otros productos y servicios para la humanidad. De aquí la obligación de promover su conservación. La apicultura es un tema de interés mundial, pero son pocos cursos los que se ofertan en universidades y organizaciones particulares que contemplan la cría de las abejas. A través del uso de tecnologías actuales y la gamificación se propone un juego didáctico que favorezca el aprendizaje en apicultura, específicamente el proceso de la cosecha de miel. El videojuego proporciona el conocimiento que se requiere para el manejo de las abejas melíferas de una manera virtual antes de llevar a los estudiantes a los escenarios reales, contribuyendo a una reducción del estrés que se puede producir en las colmenas y reduciendo el número de accidentes que pueden ocurrir.
\end{abstract}

Palabras clave: Abejas melíferas; Cosecha de miel; Enseñanza en línea; Gamificación.

\section{Introducción}

En estos últimos años se ha reportado una disminución de abejas melíferas a nivel global, esta disminución se debe a diferentes factores, pero el principal y el que une a todos los factores es la intervención humana. Esta intervención afecta en cierta medida a la capacidad de las colonias de abejas para que puedan sustentarse. Uno de los factores que ocasiona la disminución de las abejas es el estrés [1]. El estrés de las abejas es un factor que disminuye en cierta medida la vida de estas, y en ocasiones detiene el trabajo por periodos de tiempo, ya que cuando se realizan revisiones de las colmenas se rompe la dinámica que estas llevan en su interior. Una causa del estrés es la excesiva manipulación de los apicultores sobre las colmenas [2].

Castillo Pinkus., LG., Menéndez Domínguez, VH., De Araujo Freitas,

JC., Coello Cervera, E.

Universidad Autónoma de Yucatán

Mérida, México.

Email: carlos.m.a.nbs@gmail.com, mdoming@correo.uady.mx,

afreitas@correo.uady.mx, ecoello@correo.uady.mx

Moguel Ordóñez, YB.

Instituto Nacional de Investigaciones Forestales, Agrícolas y Pecuarias

Mérida, México.

Email: moguel.yolanda@inifap.gob.mx
Con el propósito de reducir la intervención humana en los procesos de enseñanza y aprendizaje relacionados con el adecuado manejo de las colmenas, la Universidad Autónoma de Yucatán desarrolló un diplomado en línea en Apicultura [3].

Hoy en día, los videojuegos y las aplicaciones de los teléfonos y tabletas se han masificado. La gamificación ha hecho que el aprendizaje sea entretenido, divertido y agradable de aprender [4]. Sacarle provecho a esta aparente dependencia que las poblaciones nativas o migrantes de la tecnología móvil tienen hoy en día, hace que resulte relevante como una estrategia de aprendizaje de temas relacionados con la apicultura.

Otro aspecto que se tomó en cuenta en el desarrollo de un videojuego de apicultura es la capacidad de simular una colmena y sus panales y así los nuevos apicultores pueden aprender procesos apícolas sin la necesidad de contar con una colmena propia o ir a una. Con esto las colmenas no acumulan estrés por el exceso de manipulación de los panales por los estudiantes de apicultura, no existen contaminaciones en las colmenas por un mal uso de los combustibles utilizados en un ahumador y otros factores que pueden causar los estudiantes de apicultura por la falta de conocimientos.

Con este videojuego los estudiantes de apicultura simularán el proceso de cosecha de miel y con ello se lograrán las siguientes competencias:

- Distinguir cuál es el combustible adecuado para el ahumador que no contamine la miel;

- Diferenciar los panales para la recolección de miel y los que tienen crías.

- Identificar los panales adecuados para la extracción y cosecha de miel

Desarrollar estas competencias ayudará a los estudiantes de apicultura a reducir los daños en las abejas, movilizarse rápido para no molestar por mucho tiempo a las abejas y causarles estrés.

Este artículo está organizado de la siguiente manera: en la próxima sección se describe el estado del arte sobre la gamificación. Posteriormente, se describe la secuencia del videojuego y la interacción del usuario. Por último, se describe el impacto esperado y las conclusiones.

\section{Estado del arte}

Prensky [4], menciona que los graduados universitarios promedio de hoy día han gastado menos de 5,000 horas de sus vidas leyendo, pero más de 10,000 horas jugando videojuegos, de tal forma que podemos emplear esta ventaja digital de la 
gamificación para que los estudiantes aprovechen recursos tecnológicos para aprender lo que antes se hacía de la manera tradicional.

La gamificación (o ludificación) se define como "el uso de estrategias, modelos, dinámicas, mecánicas y elementos propios de los juegos en contextos ajenos a éstos, con el propósito de transmitir un mensaje o unos contenidos o de cambiar un comportamiento, a través de una experiencia lúdica que propicie la motivación, la implicación y la diversión" [5].

Al convertir el proceso enseñanza-aprendizaje en una actividad lúdica los alumnos puedan divertirse mientras aprenden, dándoles motivación y compromiso de aprender, que son los problemas que se han tenido que enfrentar las escuelas [6]. Gallego-Durán et al. [7] encontraron varios catalizadores de aprendizaje en los videojuegos que se pueden utilizar para un mejor aprendizaje para las personas.

Esto lleva a que el juego que se propone ayudará a los estudiantes de apicultura a aprender de una manera diferente pero entretenida el proceso de la cosecha de miel en una colmena.

\section{Propuesta}

El juego fue desarrollado en la plataforma Unity, con código C\#, y fue creado en un ambiente 2D con un diseño basado en colores orgánicos asociados con la naturaleza. Este consiste en cuatro pasos fundamentales (Figura 1) que deben hacer los estudiantes de apicultura para cosechar la miel que han recolectado las abejas de un apiario [8]. Estos son distribuidos en tres fases.

Primera fase. El usuario deberá seleccionar el combustible correcto para el encendido de su ahumador. Los combustibles son materiales que se emplean en un ahumador para generar un humo denso y blanco con el cual se distrae el instinto natural de las abejas a aguijonear. En la pantalla inicial del videojuego (Figura 2) se mostrarán 9 posibles opciones en diferente orden de la cual el jugador seleccionará 1 , solo 4 son correctas. El usuario tiene solo 30 segundos para seleccionar el combustible adecuado; en caso, de no seleccionarlo, pasará a la siguiente fase y se le informará al jugador que no eligió un combustible. Esto hace que se le descuenten puntos.

Segunda fase. El usuario debe seleccionar los panales que contienen miel operculada. En la pantalla del videojuego (Figura 3) se presenta una colmena vacía en la parte superior izquierda y otra llena de panales de diversos tipos en la parte inferior izquierda de la misma de donde los seleccionará.

Los tipos de panales que pueden presentarse son 7: (1) Panal con polen; (2) Panal con miel operculada o madura; (3) Panal con miel desoperculada o inmadura; (4) Panal con crías abiertas; (5) Panal con cría operculada; (6) Panales vacíos; y (7) Cuadros con hojas de cera estampada.

El usuario identificará los panales adecuados para la cosecha de miel que corresponden a los que contienen miel operculada o madura seleccionándolos de la caja llena (colmena) y acomodándolos en la caja vacía. La selección de panales otorga o quita puntos según si la elección es correcta.

Si los panales de la colmena tienen crías, el estudiante deberá regresarlas y acomodarlas en el centro de una caja en la ubicación correcta (crías abiertas al centro y las operculadas a los lados de estas. En caso de que un panal no contenga crías, deberá regresarlo a la colmena y ponerlo al alza de la misma o designarlo para la cosecha si es que se trata de un panal con miel aun no operculada.

En el videojuego, los colores y formas de las celdas determinan su tipo: las celdas vacías tienen un diseño plano. Las celdas que contienen miel, polen o larvas tienen tonalidades ocre

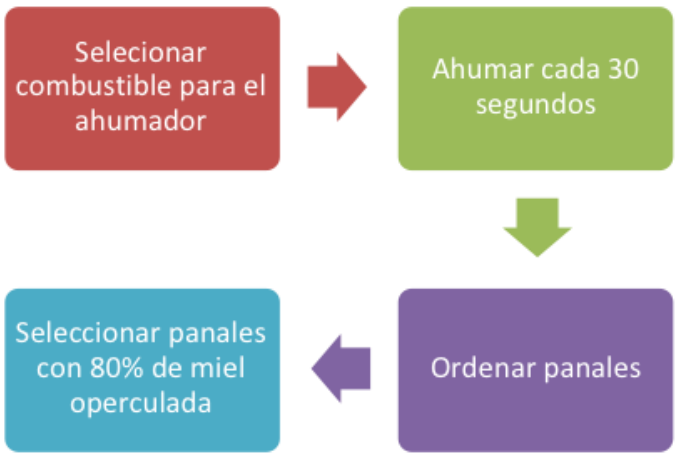

Figura 1. Proceso de cosecha de miel

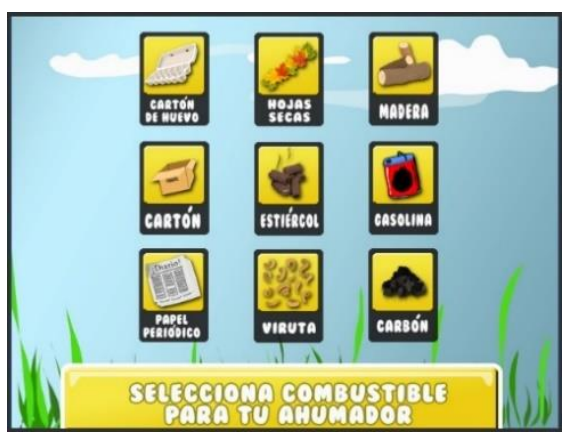

Figura 2. Elección de combustible

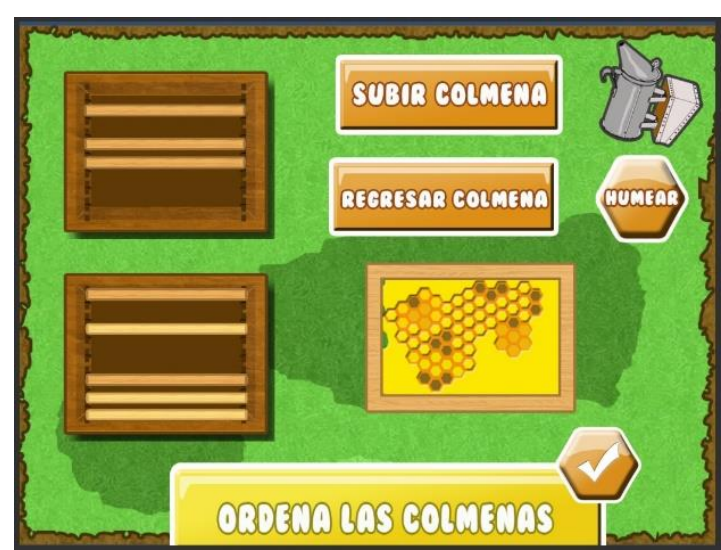

Figura 3. Segunda fase

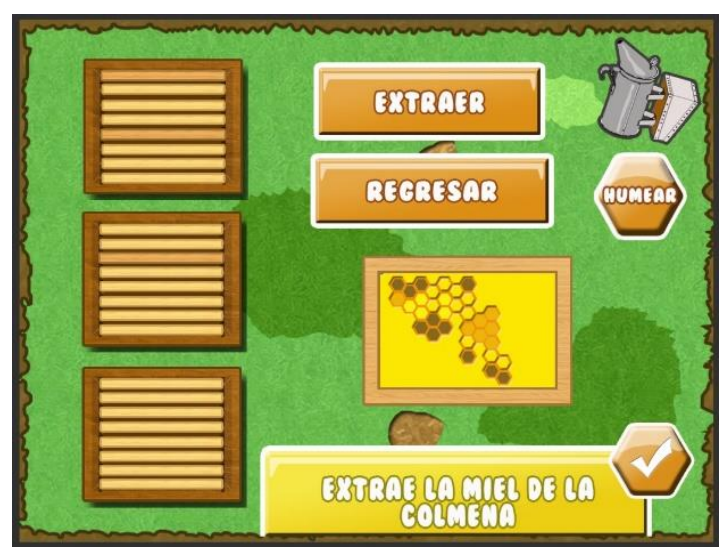

Figura 4. Tercera fase. 


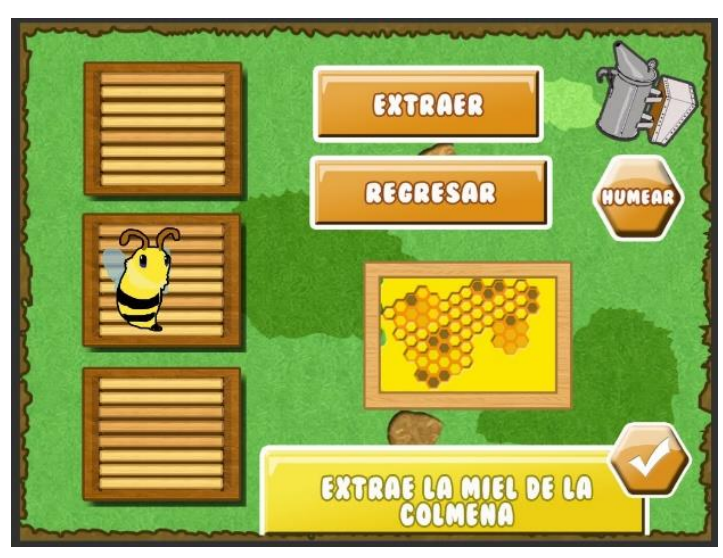

Figura 5. Aparición de abeja

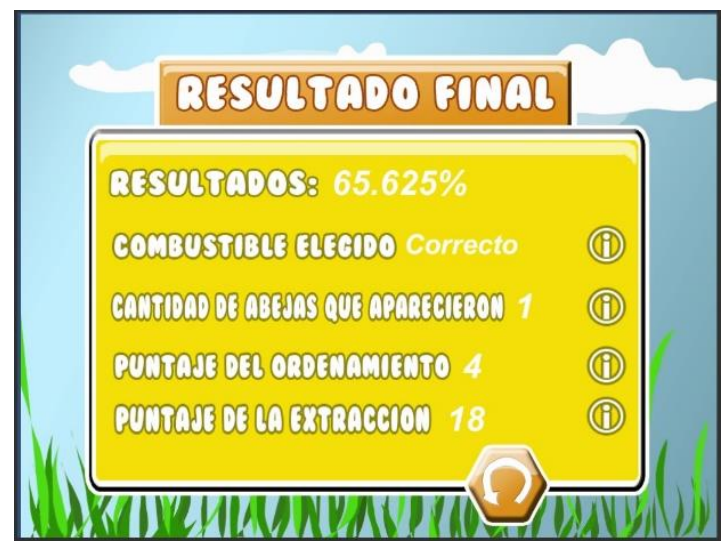

Figura 6. Pantalla de resultados

y un diseño con relieve. Al colocar el mouse sobre la celda el usuario obtiene información sobre el tipo de celda.

Tercera fase. Se le muestra al usuario tres cajas llenas de panales, las cuales deberá de inspeccionar y observar cuáles son las adecuadas para extraer miel y cuáles les falta más tiempo para alcanzar la madurez (Figura 4).

Cada vez que el usuario selecciona un panal este se le muestra en pantalla para que decida si se extrae su miel o se regresa a la colmena. En ambos casos se marca el panal revisado y se otorgan puntos si se tomó la decisión correcta. Un panal que debe ser cosechado es aquel que tiene al menos un $80 \%$ de celdas que contienen miel operculada y sin crías [8].

En las pantallas de la fase 2 y 3 del videojuego se presenta un objeto en la parte superior derecha, el ahumador, e incluye un botón "Humear". El jugador debe seleccionar este botón o el objeto a lo más cada 30 segundos durante el tiempo que esté realizado la tarea en cada fase. En el videojuego se presenta un efecto de niebla en la pantalla que dura unos segundos como forma de retroalimentar al usuario que ha humeado la colmena.

La finalidad de humear el área de trabajo es controlar el comportamiento de las abejas y evitar que estas molesten en la ejecución de la tarea del apicultor. Por cada vez que no se humea la colmena en un período de 30 segundos aparece una abeja (Figura 5) que limitan la selección de opciones al interponerse entre el usuario y los objetos. Por cada abeja que salga en la pantalla se le restan puntos al jugador.

El uso del ahumador es indispensable en el apiario pues controla el instinto de defensa de las abejas para aguijonear a los intrusos [8].
Después de haber pasado por las tres fases del juego, aparece una pantalla de resultados (Figura 6) en términos de:

- Porcentaje de desempeño

- Combustible elegido

- Abejas que aparecieron por no humear en los tiempos establecidos

- Puntaje alcanzado en la fase de ordenamiento

- Puntaje alcanzado en la fase de extracción

Para que el usuario obtenga más información de su resultado, debe presionar el ícono de información, y se presenta una ventana con la razón del resultado otorgado.

\section{Conclusiones}

Los recursos digitales son una herramienta accesible a estudiantes que tienen a bien aprender conocimientos nuevos a través de su interacción con herramientas tecnológicas en las que se les presentan retos que deben resolver mediante actividades de aprendizaje programadas en línea. Este juego desarrollado permitirá a los estudiantes aprender un manejo básico de la colmena sin estar en un apiario y no causar estrés a las abejas durante el proceso de aprendizaje. La selección de Unity como plataforma de desarrollo ha permitido que el videojuego pueda ejecutarse en cualquier sistema operativo (Windows, MacOS, IOS, Android, Web) posibilitando su utilización por cualquier usuario que tome el diplomado en línea en Apicultura de la Universidad Autónoma de Yucatán. Como trabajo futuro está evaluar la propuesta en un experimento controlado y recibir retroalimentación para la mejora de la interfaz del usuario, así como identificar el impacto del videojuego en el proceso de enseñanza-aprendizaje de cómo extraer miel de una colmena.

\section{Referencias}

[1] Valega, O. Estrés en las Abejas. Apiservices. (2018) https://www.apiservices.biz/es/articulos/orden ar-porpopularidad/1161-estres-en-las-abejas

[2] Jean-Prost, P. Apicultura: conocimiento de la abeja. Manejo de la colmena. MundiPrensa Libros (2007)

[3] Universidad Autónoma de Yucatán. Diplomado en línea en Apicultura. (2014) http://beesyucatan.blogspot.com/2014/09/dipl omado-enapicultura.html

[4] Prensky, M. Digital natives, digital immigrants. On the Horizon, Vol. 9 Issue: 5, pp.1-6, (2011) https://doi.org/10.1108/10748120110424816

[5] Gallego-Durán, F. J., Molina-Carmona, R., y Llorens-Largo, F. Gamificar una propuesta docente. Diseñando experiencias positivas de aprendizaje. (2014)

[6] J. Lee, y J. Hammer. Gamification in Education: What, How, Why Bother? Academic Exchange Quarterly, 15(2), 146 (2011).

[7] Gallego, F. J., Villagrá, C. J., Satorre, R., Compañ, P., Molina, R., y Llorens-Largo, F.. Panorámica: serious games, gamification y mucho más. ReVisión, 7(2) (2014).

[8] De Araujo, Ch., González, A., Marrufo, J. Apicultura Práctica en la Península de Yucatán. Ed UADY. (2010) 